\title{
Der neue GW-Lehrplan für die Sekundarstufe I - ministerieller Auftrag, Herausforderungen, Struktur und erste Überlegungen
}

\section{Vorbemerkungen}

Der Lehrplan für die Sekundarstufe I des Schulfaches Geographie und Wirtschaftskunde (GW) ist wortident für die Mittelschule (NMS) sowie für die Allgemeinbildende Höhere Schule (AHS) und gilt daher an beiden Schultypen. Um die Ideen des in Auftrag gegebenen neuen GW-Lehrplans besser verstehen zu können, kann ein kurzer historischer Rückblick hilfreich sein.

Mit dem Lehrplan 1985 findet eine paradigmatische Wende statt, nämlich eine Abkehr von der Schulländerkunde mit dem Prinzip vom „Nahen zum Fernen" hin zu einem lernzielorientierten, thematisch aufbauenden Lehrplan. Seitdem wird GW als doppelpoliges Zentrierfach „Geographie und Wirtschaftskunde" unter dem Gesichtspunkt der politischen Bildung verstanden. Im Zentrum stehen der Mensch und räumliche Aspekte menschlichen Handelns. Wichtige Aspekte sind folgende: Die Integration der Bereiche Geographie und Wirtschaftskunde erfolgt über das Konzept des in Raum, Gesellschaft und Wirtschaft handelnden Menschen. Dieser Lehrplan (bis 2000 als Rahmenlehrplan) ist vom Einfachen zum Komplexen thematisch gegliedert strukturiert (siehe dazu weiterführend W. Sitte 2001, S. 158 ff.; Ch. Sitte 2001, S. 223 f.), und die Wirtschaftskunde wird auf die sozialwissenschaftliche Tradition der ökonomischen Bildung orientiert (Fridrich 2018, S. 83 ff.).

Der Lehrplan 2000 führt diese Tradition weiter, ohne die inhaltliche Struktur des Lehrplans 1985 grundlegend zu verändern. Auf einer formalen Ebene werden hingegen bedeutende Veränderungen vorgenommen, wie etwa Zielstellungen ohne Lerninhaltsangaben formuliert, Kern- und Erweiterungsbereiche definiert, eine Reduktion der Themenkreise und Zielstellungen vorgenommen und Beiträge zu den Bildungsbereichen ausführlich formuliert (siehe ausführlich Fridrich 2014, S. 36 ff.). Dieser Lehrplan ist nun seit fast zwei Jahrzehnten in Verwendung, was eine Neugestaltung erforderlich macht.

\section{Ministerieller Auftrag}

Im Herbst 2018 beauftragt das Bundesministerium für Bildung, Wissenschaft und Forschung (BMBWF) unter der Leitung von Minister Heinz Faßmann eine komplette Neuerarbeitung aller Lehrpläne für die Primarstufe und für die Sekundarstufe I im Rahmen des „Pädagogikpakets“. Nach Aufforderung des BMBWF, eine GW-Arbeitsgruppe zu bilden, wird nach mehrtägigen Beratungen eine von Stefan Hinsch geleitete Gruppe bestehend aus zwei NMS-Lehrpersonen (Carina Chreiska-Höbinger, Marcel Vorage), zwei AHSLehrern (Stefan Hinsch, Paul Hofmann) sowie zwei Fachdidaktikern (Christian Fridrich, Herbert Pichler) gebildet. Mit dieser personellen Zusammensetzung soll auch die Anschlussfähigkeit des semestrierten kompetenzorientierten Lehrplans der Sekundarstufe II (2016) gewährleistet werden.

Der offizielle Start der Lehrplanarbeit wird mit einer Kick-off-Veranstaltung, zu dem die Mitglieder aller Fächer eingeladen sind, im BMBWF am 28.2. und 1.3.2019 eingeleitet. Es werden am ersten Tag zahlreiche formale und inhaltliche Vorgaben sowie Fragestellungen mithilfe umfangreicher Unterlagen präsentiert und diskutiert: Prozess zur Erarbeitung der Fachlehrpläne in der Primarstufe und Sekundarstufe I, Auftrag aus dem Pädagogikpaket, strukturgebende Elemente der Lehrpläne, reflexive Grundbildung als Basis, zentrale fachliche Konzepte, Kompetenzorientierung und Leistungsbeurteilung, Elemente des Lehrplans, Kompetenzmodelle und Kompetenzbereiche, Kompetenzbeschreibungen, Informatische Bildung, Medienbildung, Sprachliche Bildung, Politische Bildung, Wirtschafts- und Verbraucher/innenbildung, Gesamtprojekt (Überblick, Eckpunkte und Zielsetzung, Zeitrahmen, Prozess zur Erarbeitung), Arbeitsauftrag Arbeitsphase, Zeitplan und nächste Schritte etc. Am zweiten Tag diskutieren die Mitglieder/innen innerhalb der Fachgruppen. In GW wird in Adaption des Kompetenzmodells für Geographie und Wirtschaftskunde (bm:ukk 2012) ein für die Sekundarstufe I ad- 
aptiertes Kompetenzmodell skizziert. Zudem wird die Umbenennung des Faches in „Geographie und wirtschaftliche Bildung" vorgeschlagen, weil der Begriff „Wirtschaftskunde“ in vielfacher Weise veraltet erscheint und nicht die Orientierung einer lebensweltorientierten ökonomischen Bildung erkennen lässt. Weiters wird übereingekommen, das Team der sechs Mitglieder/innen zu einem erweiterten Fachteam um Thomas Jekel, Lars Keller und Alfons Koller auszubauen und die Expertise dieser drei Kollegen laufend und direkt zu integrieren. Schließlich wird festgehalten, dass der Prozess der interessierten Fachöffentlichkeit dargelegt und diskutiert werden soll, was im Rahmen der Fachdidaktiktagung in Schlierbach am 8.4.2019 abends unter reger Anteilnahme von Kolleg/ innen zum ersten Mal erfolgt. Weitere Präsentationen werden folgen, auch dieser Beitrag ist in dem Sinn zu verstehen.

\section{Chancen und Probleme des ministeriellen Auftrags im Detail}

Die Lehrplangruppe GW begrüßt die überfällige Neukonzeption der Lehrpläne der Sekundarstufe I ausdrücklich und teilt die Ziele, der Schulpraxis zukunftsorientierte und praktikable Lehrpläne zur Verfügung zu stellen. Auch die verpflichtende Einbindung von Fachdidaktiker/innen, Schulpraktiker/innen sowie die geforderte Einbindung der Fachwissenschaft in den Entwicklungsprozess sind als wesentliche Elemente der Qualitätssicherung positiv hervorzuheben.

Im Rahmen der Auftaktveranstaltung vom 28.2. bis 1.3.2019 sind jedoch mehrere gravierende konzeptionelle Probleme des Projektes „Lehrpläne 2020“ bzw. des zu Grunde liegenden politischen Auftrags offen zu Tage getreten, die eine Zielerreichung erschweren:

1. Der politisch gesetzte enge Zeitrahmen erschwert einen qualitativ hochwertigen Entwicklungsprozess innerhalb der Arbeitsgruppen sowie einen mehrstufigen Rückmeldeprozess durch Schulpraktiker/innen. Weiters wird die bei jedem Lehrplan bisher fehlende Koordination und Kooperation zwischen den Lehrplangruppen der einzelnen Fächer (Querschnittsmaterien, Unterrichtsprinzipien, übergreifende Themen) dadurch erschwert bis verunmöglicht. Die dafür im Projektplan vorgesehenen punktuellen Treffen der Lehrplangruppenleiter/innen können dies nur unzureichend abfedern.

2. Zum Zeitpunkt der Auftragserteilung bleibt ähnlich wie bei der Erstellung des semestrierten Lehrplans der SII (2016) der für die gesamte Erstellungsarbeit des Lehrplans zentrale Kompetenzbegriff weitgehend undefiniert. Un- terschiedliche Kompetenzbegriffe, Termini wie Kompetenzbereiche, Kompetenzbeschreibungen, Mindest- und Grundanforderungen etc. wurden im Rahmen des Kick-Offs in Inputvorträgen, Erläuterungen, Fragebeantwortungen sowie in den Handreichungen widersprüchlich verwendet. Erst am 25. April 2019 erfolgt in einer internen Stellungnahme des BMBWF an die Lehrplangruppen eine Festlegung auf die umfassende Kompetenzdefinition nach Weinert, die aber im Text nachfolgend sofort wieder aufgeschnürt und dadurch für einzelne Fachgruppen unterschiedlich interpretierbar und umsetzbar wird.

3. Ein zentraler Bestandteil der neuen Fachlehrpläne sollen fachspezifische Kompetenzstrukturmodelle sein. Zahlreiche Wortmeldungen aus den Fachgruppen wiesen darauf hin, dass es für kaum ein Fach belastbare, fachwissenschaftlich und fachdidaktisch abgesicherte Kompetenzmodelle gibt, die vorhandenen Modelle meist international höchst umstritten sind und daher nicht einfach übernommen werden können.

4. Aus Sicht der Fachgruppe GW ist die Einführung zentraler fachlicher Konzepte in den neuen Fachlehrplänen zu begrüßen, die Lehrplangruppe hat bereits im semestrierten Lehrplan der Sekundarstufe II (2016) vergleichbare Basiskonzepte eingeführt und darüber hinaus in einem Sonderheft der Zeitschrift GW-Unterricht 147/2017 dokumentiert und erläutert.

5. Im Rahmen des Kick-Offs erfolgte die begrüßenswerte Vorgabe eines Bekenntnisses zur reflexiven Grundbildung. Es behindert jedoch einen effektiven Arbeitsprozess der Lehrplangruppen im engen Zeitkorsett, dass zum Start der Arbeit der Lehrplangruppen der Allgemeine Teil des Lehrplans nicht einmal in Grundzügen vorhanden ist. Vor allem auch, weil die Lehrplangruppen den Auftrag bekommen haben, Verdoppelungen mit dem Allgemeinen Teil zu vermeiden. Der Allgemeine Teil des Lehrplans liegt zum Zeitpunkt des Verfassens dieser Zeilen noch immer nicht vor. ${ }^{1}$

6. Die Entscheidung, die konzeptionell völlig unpassende Terminologie "Lehrstoff" nicht mehr in kompetenzorientierten Lehrplänen zu verwenden, ist zu begrüßen. Problematisch erschien eingangs das dafür eingeführte Strukturelement des Lehrplans „Anwendungsbereiche“. Die Anwendungsbereiche sollten „den Kompetenzerwerb unterstützen“ und „weisen repräsentativen und exemplarischen Charakter auf". Die Formulierung ,verbindliche Themen und Inhalte, die im Unterricht behandelt werden müssen" steht je-

Stand: 30.5.2019 
doch im krassen Widerspruch zur Intention von Kompetenzmodellen, gängigen Kompetenzdefinitionen und des kompetenzorientierten Unterrichts. Die Konsequenzen dieser Vorgabe hätten konzeptionell einen Rückschritt vor die lernzielorientierten Lehrpläne bedeutet. Von Seiten des BMBWF wurde die deutliche Kritik an dieser Vorgabe gehört und die entsprechenden Vorgaben wurden entschärft, die Anwendungsbereiche sind nun optional, wenn die verbindlich zu unterrichtenden Inhalte bereits über die Kompetenzbeschreibungen ausreichend abgedeckt sind.

7. Die einseitige Hervorhebung von sechs bisherigen Unterrichtsprinzipien $\mathrm{zu}$ verpflichtend in mindestens fünf Fachlehrplänen zu verankernden übergreifenden Themen, könnte eine problematische sachliche Schieflage der neuen Lehrpläne erzeugen. „Informatische Bildung, Medienbildung, Sprachliche Bildung, Politische Bildung und Wirtschaftsbildung" (Zitat Unterlagen und Foliensatz zum Kick-Off) sind unbestritten wichtig. Es ist auch zu begrüßen, dass die „Umweltbildung für nachhaltige Entwicklung", durch den politischen Druck der Klimaproteste, die ursprünglich nur fünf Unterrichtsprinzipien um ein sechstes ergänzt. Und es ist auch sinnvoll, bisherige Unterrichtsprinzipien, für die formal alle, praktisch aber oft niemand zuständig war, fix in den Fachlehrplänen zu verankern. Für GW sind dabei Sprachliche Bildung, Informatische Bildung, Politische Bildung und Umweltbildung vorgesehen.

Es erscheint jedoch höchst bedenklich, dass angesichts der virulenten globalen und gesellschaftlichen Herausforderungen Unterrichtsprinzipien wie beispielsweise Interkulturelles Lernen oder Erziehung zur Gleichstellung von Frauen und Männern nicht zu übergreifenden Themen erklärt werden, die sich in Lehrplänen konkret ausdrücken müssen. Dies ist sachlogisch, fachwissenschaftlich, gesellschaftspolitisch und in Bezug auf die Gegenwarts- und Zukunftsbedeutung nicht erklärbar.

8. Ausnahmslos Kritik aller Fachgruppen hat die Ankündigung ausgelöst, dass der Lehrplan vor der finalen Überarbeitung und damit vor der öffentlichen Begutachtung "der Wirtschaft zur Kontrolle" (Zitat im Rahmen des Kick-Offs) vorgelegt werde.

9. Schließlich ist auch die Absicht in einem zweiten Projektschritt Kompetenzraster zur Leistungsbewertung und -beurteilung zu erstellen, kritisch zu prüfen. Es ist zu begrüßen, dass im Zusammenhang mit einer zukünftigen neuen LBVO über Wege nachgedacht wird, den Lern- prozess im Sinne einer förderlichen Leistungsbewertung nicht permanent durch punktuelles summatives Prüfen und Benoten zu behindern, es zu einer laufenden Dokumentation von Schüler/innenleistungen kommen soll und erst im Gesamten zu einer Benotung. Die Erstellung und der Einsatz der Kompetenzraster selbst bedeutet jedoch unserer Einschätzung nach die größte „Falle“ im Rahmen des Projektes Lehrplan 2020: Wiederholt hat der ob seiner Expertise geschätzte Kollege Neuweg (wissenschaftliche Begleitung) selbst darauf hingewiesen, dass (auch im abgelaufenen Pilotprojekt des Ministeriums) bisher keine belastbaren und praktikablen Kompetenzraster zur Leistungsbewertung und -beurteilung erstellt werden konnten. Die von ihm gezeigten prototypischen Beispiele funktionieren bislang nur in der Theorie, jedes gezeigte konkrete Beispiel erfüllte die von ihm selbst formulierten Ansprüche nicht.

$\mathrm{Zu}$ den Herausforderungen des ministeriellen Arbeitsauftrags kann zusammenfassend festgehalten werden, dass auf fachlich und fachdidaktisch begründete Kritik von Seiten des BMBWF bereits in einigen Punkten zielorientiert und pragmatisch reagiert worden ist. Wie eben aufgelistet, sind einige Kritikpunkte noch nicht ausreichend ausgeräumt, wir setzen aber weiter auf die bislang gute Gesprächsbasis und einen fortlaufenden Diskussionsprozess.

\section{Weitere Vorgangsweise bis Juni 2019}

Am 28.3.2019 findet nach umfangreichen Vorarbeiten und Austausch von Diskussionspapieren zur Lehrplanentwicklung das erste Treffen des erweiterten Fachteams an der Universität Salzburg statt. Bereits zu Beginn zeigt sich die Schwierigkeit der ministeriellen Forderung von zielgruppenspezifischen Formulierungen (für Lehrpersonen und ggf. auch für Schüler/innen) einerseits und einer korrekten fachdidaktischen Formulierung andererseits. An Textvorlagen wie dem Positionspapier Sozioökonomische Bildung, dem fachdidaktischen Grundkonsens 2.0, dem Grundsatzerlass für die Wirtschafts- und Verbaucher/innenbildung etc. orientiert sich Christian Fridrich bei der Erstellung eines Grundlagentext für eine erste Version der Bildungs- und Lehraufgaben, der didaktischen Grundsätze und des Kompetenzmodell des Faches, die ausführlich diskutiert und gemeinsam ausformuliert wird. Aufträge zur Weiterarbeit an den didaktischen Grundsätzen gehen an Paul Hofmann und Herbert Pichler. Christian Fridrich und Stefan Hinsch erhalten die Aufgabe, Grundlagen für die zentralen fachlichen Konzepte zu erarbeiten. 
Beim zweiten Treffen des erweiterten Fachteams am Schulzentrum Ungargasse in Wien am 24.4.2019 werden Adaptierungen der bisherigen Texte sowie eine Fixierung einer ersten Version der didaktischen Grundsätze und weiter Teile der fachlichen Konzepte vorgenommen. Es wird eine dislozierte Nacharbeit an den fachlichen Konzepten und Fertigstellung für die erste Feedback-Runde gewünscht. Das Feedback über bereits vorliegende Texte wird eingeholt: Paul Hofmann von der AHSBundesarge GW, Alfons Koller von Linzer Kolleg/ innen, Marcel Vorage von der NMS Bergheim, Carina Chreiska-Höbinger von der Praxismittelschule der PH Wien. Zudem geben wichtige Stakeholder wie etwa Vertreter/innen von der Arbeiterkammer Wien, der Industriellenvereinigung, der Wirtschaftskammer Wien und der Oesterreichischen Nationalbank Stellungnahmen ab. Die dadurch gewonnen Impulse und Rückmeldungen werden in einem weiteren Überarbeitungsschritt diskutiert und gegebenenfalls eingearbeitet werden.

Im Zuge des dritten Treffens des erweiterten Fachteams am Schulzentrum Ungargasse in Wien am 13.5.2019 wird ein Input von Lars Keller zur Integration von Sustainable Development Goals geleistet, und es ergeht ein Überarbeitungsauftrag der bisherigen Texte an diesen Kollegen. Zudem wird der Prozess der Formulierung der Kompetenzbereiche und potenzieller Kompetenzen der einzelnen Klassen gestartet.

\section{Künftige Meilensteine der Lehrplanarbeit}

Bis zum 15. September 2019 muss ein Vorschlag für den gesamten Lehrplan vorliegen, der Mitte Oktober mit den Verantwortlichen des Ministeriums besprochen wird. Feedback von Stakeholdern, Kolleginnen und Kollegen sowie des Bildungsministeriums wird in weiteren Schritten bis Februar 2020 eingearbeitet. Dann verlässt der Lehrplan die Arbeitsgruppen Richtung Begutachtung und Gesetzgebung und sollte im September 2020 kundgemacht werden. Anschließend folgt eine Frist der Anpassung für Schulbuchverlage und Fortbildungen. Ab dem Schuljahr 2022/23 wird der neue Lehrplan dann in der 5. Schulstufe umgesetzt. Ob der Zeitplan so hält oder durch politische Turbulenzen ein wenig anders verläuft, kann natürlich niemand garantieren.

Der Prozess der Lehrplanentwicklung wird auf einer Web-Seite auf der Lernplattform $G W$ begleitet: http://gweduhi.at/LP21GW. Dort ist die jeweils aktuelle Version abrufbar. Alle interessierten Leserinnen und Leser sind eingeladen, ihre Kommentare und konkreten Textvorschläge dort abzugeben.

Nach einer Vorstellung der ersten Entwürfe auf der Tagung „Zukunft Fachdidaktik GW“ am 8.4.2019 und einer ersten Feedbackrunde im April 2019 soll am IMST-Fachdidaktiktag, dem Mittwoch, 25.9.2019, in Klagenfurt der Diskussionsprozess fortgeführt werden. Alle sind zu dieser Lehrer/innenfortbildung sehr herzlich eingeladen; die Anmeldung ist bis 15.9.2019 über PH-online der PH-Linz möglich.

\section{Erstversion bereits vorliegender Texte zur Diskussion}

\section{Geographie und Wirtschaftliche Bildung}

\section{Bildungs- und Lehraufgabe}

Im Mittelpunkt des Unterrichtsgegenstandes Geographie und Wirtschaftliche Bildung (GW) steht der handelnde Mensch in Gesellschaft, Wirtschaft, Politik und Umwelt. Die Zusammenhänge zwischen diesen Bereichen werden aus räumlicher und ökonomischer Perspektive erschlossen. Ein wichtiger Ausgangspunkt sind dabei die Lernenden und ihre Lebenswelten.

Im kompetenzorientierten GW-Unterricht werden in allen Anforderungsbereichen (Reproduktion - Transfer - Reflexion, Bewertung und Problemlösen) zentrale geographische und wirtschaftliche Inhalte, Konzepte und Methoden vermittelt und erarbeitet. Diese orientieren sich an aktuellen fachlichen und fachdidaktischen Erkenntnissen.

Unterrichtsrelevante räumliche und ökonomische Prozesse sowie ihre Darstellung sind gesellschaftlich eingebettet. Sie sollen für Schülerinnen und Schüler als gestaltbar und veränderbar erfahren werden.

\section{Angestrebte Ziele des Unterrichts im Unterrichtsgegenstand Geographie und Wirtschaftliche Bildung am Ende der 8. Schulstufe}

Junge Menschen sollen befähigt werden, sich in unserer ökonomisch, technologisch und durch Digitalisierung geprägten Gesellschaft zu orientieren, sich eine fachlich begründete, demokratisch und ethisch fundierte Mei- 
nung zu bilden und diese zum Ausdruck zu bringen. Sie sollen altersgemäß an Prozessen in Gesellschaft, Wirtschaft, Politik und Umwelt mündig und verantwortungsbewusst mitwirken.

Schülerinnen und Schüler sollen in den komplexen Lebenswelten durch Analyse und Reflexion ihre individuelle Orientierungs-, Urteils- und Handlungsfähigkeit entwickeln. Besondere Bedeutung hat dabei die Auseinandersetzung mit den Bereichen Haushalt, Konsum, Geld, Arbeitswelt, naturräumliche und sozialgeographische Prozesse, Mensch-Umwelt-Beziehungen, Nachhaltigkeit und globaler Wandel.

Damit trägt der GW-Unterricht dazu bei, im privaten, beruflichen und öffentlichen Bereich reflektiert handeln zu können.

\section{Kompetenzmodell des Unterrichtsgegenstandes Geographie und Wirtschaftliche Bildung}

Ziel des GW-Unterrichts ist die Förderung und Entfaltung des mündigen und reflektierten jungen Menschen, der im Wirkungsgefüge „Gesellschaft-Wirtschaft-Politik-Umwelt“ handlungsfähig ist. Dazu sind fachlich fundierte Inhalte, Konzepte und Methoden notwendig, die die Entwicklung von Orientierungs-, Urteils- und Handlungskompetenz unterstützen.

Orientierungskompetenz umfasst das Wahrnehmen, Verstehen und Analysieren von gesellschaftlichen, wirtschaftlichen, politischen sowie umweltbezogenen Strukturen, Prozessen, Kontroversen, Konflikten und deren Darstellungen.

Urteilskompetenz bedeutet ein differenziertes, mehrperspektivisches Reflektieren und Bewerten der eigenen und gesellschaftlichen Handlungsoptionen, Handlungen und deren Folgen im Hinblick auf eine humane und nachhaltige Lebenswelt.

Handlungskompetenz basiert auf fundiertem Wissen, erlernten Fähigkeiten, gemachten Erfahrungen und entwickelten Einstellungen. Getroffene Entscheidungen sollen kommuniziert, argumentiert und wenn möglich umgesetzt werden.

\section{Didaktische Grundsätze}

Der Kompetenzerwerb im Unterrichtsgegenstand Geographie und Wirtschaftliche Bildung erfolgt auf Basis folgender Prinzipien:

- Orientierung an der Lebenswelt der Schülerinnen und Schüler durch den Bezug auf deren gesellschaftlich, wirtschaftlich und politisch geprägte Lebenswelten.

- Aktualitäts- und Zukunftsbezug durch exemplarische bildungsrelevante Themen mit Problemorientierung und -bezug.

- Kompetenz- und Handlungsorientierung durch das Verstehen von Zusammenhängen, Anwenden des Gelernten sowie Reflektieren der eigenen Einstellungen, Entscheidungen und Handlungen.

- Individualisierung durch angemessene, vielfältige und differenzierte Lernprozesse, die auf den Vorerfahrungen der Lernenden aufbauen.

- Mehrperspektivität und Pluralität durch unterschiedliche fachliche Zugänge, individuelle Sichtweisen sowie verschiedene Welt- und Menschenbilder.

- Wissenschaftsorientierung durch altersgemäße Berücksichtigung aktueller Erkenntnisse der wissenschaftlichen Geographie und der Sozial- und Wirtschaftswissenschaften.

Der GW-Unterricht leistet einen besonderen Beitrag zu folgenden „übergreifenden Themen“:

- Bildung für nachhaltige Entwicklung durch Berücksichtigung räumlicher, ökologischer, sozialer und wirtschaftlicher Perspektiven.

- Politische Bildung durch die Auseinandersetzung mit kontroversen Interessen, Wahrnehmungen und Darstellungen sowie mit Konflikten, Macht und Aushandlungsprozessen.

- Berufsorientierung bietet den Lernenden Unterstützung bei der Auslotung ihrer Interessen und Fähigkeiten für weiterführende Bildungswege und eine spätere Berufswahl.

Darüber hinaus sind folgende fachdidaktische Leitgedanken zu berücksichtigen:

- Alle Zielstellungen und Inhalte des GW-Unterrichts sollen im Wirkungsgefüge "Gesellschaft - Politik Wirtschaft - Umwelt" bearbeitet werden.

- Dabei sollen Fallbeispiele auf unterschiedlichen Maßstabsebenen (persönlich, lokal, regional, national und global) erarbeitet werden. 
- Der GW-Unterricht ist den Werten eines ökologisch, ökonomisch und sozial nachhaltigen Lebens, der Demokratie, den Menschenrechten, dem sozialen Ausgleich sowie der Toleranz verpflichtet.

- Die Nutzung digitaler (Geo-)Medien erfolgt in kompetenter, kritischer und kreativer Weise zum Erschließen, Produzieren und Kommunizieren von geographischen und wirtschaftlichen Inhalten.

- Die kontinuierliche regionale Zuordnung der Fallbeispiele unterstützt den Aufbau eines topographischen Grundgerüstes. Dies erfolgt ausschließlich im Rahmen von sinnvollen inhaltlichen Zusammenhängen.

- Originale Begegnungen an außerschulischen Lernorten stellen einen wesentlichen Bestandteil des GWUnterrichts dar.

- Mit aufsteigender Schulstufe werden Aufgabenstellungen zunehmender inhaltlicher und sprachlicher Komplexität gewählt.

\section{Zentrale fachliche Konzepte}

Folgende zentrale fachliche Konzepte sollen im GW-Unterricht an geeigneten Fallbeispielen veranschaulicht und vermittelt werden.

\section{Gemeinsamkeiten und Unterschiede}

Kinder und Jugendliche erfassen menschliches Leben und Wirtschaften über Aspekte des Gemeinsamen und Unterschiedlichen, indem Bezüge zu Bekanntem gebildet werden. Wahrnehmung und Darstellung setzen das Erkennen von Differenzen voraus. Vielfalt (Diversität) und Ungleichheit (Disparität) sind im Wirkungsgefüge „Gesellschaft - Wirtschaft - Politik - Umwelt" erschließbar.

\section{Vernetzung und Veränderung}

Menschen leben und wirtschaften nicht allein, sondern vernetzt. Daher haben menschliche Handlungen an einem Ort oft Auswirkungen auf andere, manchmal weit entfernte Orte und Menschen. Vernetzung und Veränderung sind damit miteinander verbunden - in den Bereichen Gesellschaft, Wirtschaft, aber auch in Mensch-Umwelt Beziehungen. Beispielsweise werden zahlreiche wirtschaftliche Zusammenhänge durch Vernetzung erklärbar, etwa Ströme von Gütern, Dienstleistungen, Informationen und Geld. Dies geschieht sowohl in Marktbeziehungen (z. B. Arbeitsmarkt und Gütermärkte) als auch durch marktferne wirtschaftliche Aktivitäten (z. B. unbezahlte Pflege und Erziehung im Haushalt).

\section{Interessen und Macht}

Menschen, Gruppen und Institutionen haben unterschiedliche, manchmal widersprüchliche Interessen. Sie können diese aber nicht in gleichem Ausmaß durchsetzen. Durch unterschiedliche Macht werden ihre Handlungsspielräume erweitert oder eingeschränkt. Dies betrifft gesellschaftliche Einschluss- und Ausschlussprozesse, die Beteiligung an Entscheidungen, den Zugang zu Ressourcen sowie Verteilungsfragen. So werden beispielsweise politische Entscheidungs $\neg$ prozesse transparent und erfahrbar.

\section{Werte und Identitäten}

Die Identitäten von Menschen bilden sich in direkten oder medialen Begegnungen in der Gesellschaft, dadurch entstehen gemeinsame Werte und Zugehörigkeitsgefühle. Menschen können sich dabei mehrfach zugehörig fühlen und mehrere Identitäten haben. Das Erkennen von Zugehörigkeiten, der Bausteine der eigenen Identitäten sowie der damit verbundenen Werte kann etwa am Beispiel nationaler Identitäten erfolgen oder an Identitätsbildung über Arbeit oder Konsum. Erkenntnisse, Urteile und Handlungen sind häufig von Werten und Interessen geleitet, die einander auch widersprechen können.

\section{Maßstabsebenen}

Analysen und Darstellungen haben immer einen bestimmten Maßstab. Dabei sind räumliche Darstellungen von der lokalen über die regionale zur globalen Ebene möglich. Auch soziale und ökonomische Sachverhalte können auf unterschiedlichen Maßstabsebenen dargestellt werden, so können soziale Interaktionen auf der Ebene Einzelner oder jener unterschiedlicher Gruppen betrachtet werden. Durch die Wahl verschiedener Maßstabsebenen kann Mehrperspektivität unterstützt und können Bewertungen und Handlungen besser begründet werden. 


\section{Acknowledgement}

Diese Publikation wurde aus Mitteln des Open-AccessPublikationsfonds der Universität Wien kofinanziert.

\section{Literatur}

Fridrich, C. (2014): Orientation towards pupils and their everyday lives, including the development of economic competences within the subject area "Geography and Economic Education" at Austrian schools. In: Osuch, W. \&, B. Wójtowicz. (Hrsg.): Annales Universitatis Paedagogicae Cracoviensis 162, Studia Geographica VI. Kraków: Wydawnictwo Naukowe Uniwersytetu Pedagogicznego w Krakowie. S. 33-52.

Fridrich, C. (2018): Sozioökonomische Bildung an allgemeinbildenden Schulen der Sekundarstufe I und II in Österreich. Entwicklungslinien, Umsetzungspraxis und
Plädoyer für das Integrationsfach Geographie und Wirtschaftskunde. In: Engartner, T., C. Fridrich, S. Graupe, R. Hedtke \& G. Tafner (Hrsg.): Sozioökonomische Bildung und Wissenschaft. Entwicklungslinien und Perspektiven. Wiesbaden: Springer. S. 81-108.

Sitte, Ch. (2001): Der GW-Lehrplan 1985/86 - Neue Zielsetzungen und Inhalte. In: Sitte, W. \& H. Wohlschlägl (Hrsg.): Beiträge zur Didaktik des „Geographie und Wirtschaftskunde"-Unterrichts (= Materialien zur Didaktik der Geographie und Wirtschaftskunde, Band 16). Wien: Institut für Geographie und Regionalforschung der Universität Wien. S. 223-247.

Sitte, W. (2001): Geographie und Wirtschaftskunde (GW) - Entwicklung und Konzept des Unterrichtsfachs. In: W. Sitte \& H. Wohlschlägl (Hrsg.): Beiträge zur Didaktik des "Geographie und Wirtschaftskunde“-Unterrichts (= Materialien zur Didaktik der Geographie und Wirtschaftskunde, Band 16). Wien: Institut für Geographie und Regionalforschung der Universität Wien. S. 157-169. 